INPLASY

PROTOCOL

To cite: Hou et al. Efficacy and safety of TCM bloodletting in acute tonsillitis, A protocol for systematic review and metaanalysis. Inplasy protocol 2021120100. doi:

10.37766/inplasy2021.12.0100

Received: 22 December 2021

Published: 22 December 2021

Corresponding author: Yi Hou

hy1994315@163.com

Author Affiliation:

Shandong University of

Traditional Chinese Medicine.

Support: Shandong University of TCM.

Review Stage at time of this submission: Preliminary

searches.

Conflicts of interest:

None declared.

\section{Efficacy and safety of TCM bloodletting in acute tonsillitis, A protocol for systematic review and meta-analysis}

Hou, Y1; Wang, X2; Dong, T3; Li, X4; Yang, J5.

Review question / Objective: The purpose of this metaanalysis is to provide some possible evidence for the effectiveness of TCM bloodletting in the treatment of acute suppurative tonsillitis, so as to provide an alternative therapy plan for patients with acute suppurative tonsillitis and to compensate for the disadvantages of antibiotic therapy and tonsillectomy.

Condition being studied: A systematic evaluation of the effect of TCM bloodletting on acute tonsillitis.

Eligibility criteria: Inclusion criteria: 1.Research types.All randomized controlled trials reported in English or Chinese will be eligible forinclusion. 2.Participant types.The participants of the study are patients diagnosed as"acute tonsillitis"; regardless of age, gender, andrace; Exclusion criteria: 1.Patients with non- tonsillitis will be excluded. 2.Patients with acute tonsillitis accompanied with other symptoms will be excluded.3.If the selected articles are abstracts, letters, case reports, reviews, or nonclinical studies, they will all be excluded. 4.Articles that reuse data will be excluded.

INPLASY registration number: This protocol was registered with the International Platform of Registered Systematic Review and Meta-Analysis Protocols (INPLASY) on 22 December 2021 and was last updated on 22 December 2021 (registration number INPLASY2021120100).

\section{INTRODUCTION}

Review question / Objective: The purpose of this meta-analysis is to provide some possible evidence for the effectiveness of
TCM bloodletting in the treatment of acute suppurative tonsillitis, so as to provide an alternative therapy plan for patients with acute suppurative tonsillitis and to 
compensate for the disadvantages of antibiotic therapy and tonsillectomy.

Condition being studied: A systematic evaluation of the effect of TCM bloodletting on acute tonsillitis.

\section{METHODS}

Participant or population: Patients with acute tonsillitis.

Intervention: The intervention measures in the experimental group should contain TCM bloodlettingmoxibustion.

Comparator: As included in eligible randomized clinical trials and retrospective cohort studies.

Study designs to be included: Randomized clinical trials and retrospective cohort studies will be included.

Eligibility criteria: Inclusion criteria: 1.Research types.All randomized controlled trials reported in English or Chinese will be eligible forinclusion. 2.Participant types.The participants of the study are patients diagnosed as"acute tonsillitis"; regardless of age, gender, andrace; Exclusion criteria: 1.Patients with non- tonsillitis will be excluded. 2.Patients with acute tonsillitis accompanied with other symptoms will be excluded.3.If the selected articles are abstracts, letters, case reports, reviews, or nonclinical studies, they will all be excluded. 4.Articles that reuse data will be excluded.

Information sources: Two researchers will use standardized tables to independently extract data in duplicate from all eligible trials according to the inclusion and exclusion criteria mentioned above. In case of disagreement, they will agree through discussion, or submit the issue to a third party for evaluation.Before the screening process, the third party will use a standardized screening form, and will perform calibration exercises. Two evaluators will independently extract data according to the pre-designed extraction table, and the extracted content will include: 1. Basic information: research number, title and author of the literature, publication time, source of the literature. 2. Research objects: patient age, gender, number of trials in each group, trial time, and baseline comparability. 3. Intervention measures: different intervention measures adopted by the experimental group and the control group. 4. Research results: the result indicators needed for this systematic review, and other indicators mentioned in the article that are not needed in this systematic review.

Main outcome(s): The main observation indicators include the effective rate of TCM bloodletting, the antipyretic time, and the white blood cell count.

Quality assessment / Risk of bias analysis: The methodological quality of each included study will be assessed independently by two reviewers using two tools. The Cochrane collaboration tool will be used to assess the quality of randomized controlled trials. It comprises the following 7 aspects: random sequence generation, allocation concealment, blind method, incomplete result data, selective reporting, and other biases. The quality assessment results of each item can be divided into three grades: "low risk", "high risk" and "unclear". The more rigorous the design and the higher the methodological quality of each RCT, the lower the risk coefficient.The Newcastle Ottawa Scale (NOS) will be used to assess the quality of retrospective studies. This method includes three aspects to the evaluation: the selection method, comparability and contact exposure assessment method of case group and control group. The higher the score, the higher the quality of the study. When necessary, the consensus on this issue will be studied with the help of a third party.

Strategy of data synthesis: The RevMan5.3 software provided by the Cochrane website will be used for the analysis. Categorical variables will be expressed by odds ratio (OR) and marked with $95 \%$ confidence interval $(\mathrm{Cl})$. Continuous variables will be expressed by mean difference (MD) and 
marked with $95 \%$ Cl. If $\mathrm{P} 0.1$ or $\mathrm{I}^{2}<50 \%$, it means that the heterogeneity between groups is small, and the fixed effect model will, in this case, be used for combined analysis; when $\mathrm{P} 50 \%$, it shows that the heterogeneity between the groups is large, and the random effects model will then be used for combined analysis, and the results shown in forest plots.Analysis of potential publication bias will be shown in a funnel chart, and sensitivity analysis and subgroup analysis will be used when necessary.

Subgroup analysis: Subgroup analysis will be used to evaluate the therapeutic effects among different drugs. Inverted funnel plots and Egger's regression test will be used to determine publication bias when the number of included studies exceeds 10 in the network meta-analysis.

Sensitivity analysis: There are two methods used in sensitivity analysis, one is to change the analysis model and the other is to exclude articles one by one. An analysis model is chosen based on thel2value. Ifl2value is $>50 \%$, the random-effect model is used. Ifl2is $<50 \%$, the fixed-effect model is used. When the articles are excluded one by one and there is a change in heterogeneity after removing one article, then this article may be the source of the heterogeneity.

Country(ies) involved: China.

Keywords: bloodletting, meta-analysis, protocol, acute tonsillitis.

Contributions of each author:

Author 1 - Yi Hou.

Author 2 - Xingxin Wang.

Author 3 - Tiantian Dong.

Author 4 - Xuhao Li.

Author 5 - Jiguo Yang. 